Originalien

Chirurg 2021 · 92:49-61

https://doi.org/10.1007/s00104-020-01192-w

Online publiziert: 19. Mai 2020

(c) Der/die Autor(en) 2020
Christian Beltzer ${ }^{1}$ Markus Quante ${ }^{1} \cdot$ Myriam Rheinberger $^{1} \cdot$ Hideo Andreas Baba ${ }^{2}$. Fuat Saner ${ }^{3} \cdot$ Falko Fend ${ }^{4} \cdot$ Thomas Biet $^{5} \cdot$ Alfred Königsrainer ${ }^{1} \cdot$ Silvio Nadalin ${ }^{1}$

'Klinik für Allgemeine, Viszeral- und Transplantationschirurgie, Universitätsklinikum Tübingen, Tübingen, Deutschland

${ }^{2}$ Institut für Pathologie, Universitätsklinikum Essen, Essen, Deutschland

${ }^{3}$ Klinik für Allgemein-, Viszeral- und Transplantationschirurgie, Universitätsklinikum Essen, Essen, Deutschland

${ }^{4}$ Institut für Pathologie und Neuropathologie, Universitätsklinikum Tübingen, Tübingen, Deutschland

${ }^{5}$ Deutsche Stiftung Organtransplantation, Frankfurt am Main, Deutschland

\section{Perkutane Leberbiopsie vor Organentnahme - Einfluss auf Organallokation und Kosten in der Lebertransplantation}

plantaten aus ECD $>50 \%$ [3], und es wird vermutet, dass er in Zukunft weiter ansteigen wird.

Funktion und Langzeitüberleben nach Lebertransplantation hängen stark von der Qualität des Spenderorgans $\mathrm{ab}$, weshalb eine sorgfältige Bewertung und Auswahl desselben unerlässlich ist [4, 5]. Zur Einschätzung des Transplantatversagens in Abhängigkeit von den Spendereigenschaften haben Feng und Kollegen 2006 den „donor risk index“ (DRI) eingeführt [6], der 2012 auf die Eurotransplant-Region (ETDRI) adaptiert wurde [7]. Innerhalb der Eurotransplantat-Region ist der durchschnittliche „MELD-Score“ („model of end stage liver disease“) der Empfänger zum Zeitpunkt der Transplantation nach Einführung des MELD-basierten Zuweisungssystems von 25 auf 34 ge- stiegen. Gleichzeitig hat die Qualität der Spenderorgane abgenommen, mit $>60 \%$ suboptimalen Organen mit einem DRI $>1,5$ Punkte. Dies hat zu schlechteren Ergebnissen und zu einer verminderten Überlebensrate nach Lebertransplantation (LT) geführt [8].

Parallel zur weltweiten Zunahme der Adipositas ist die Fettleberkrankheit („fatty liver diseaese“, FLD) zu einem bedeutenden Problem bei Lebertransplantationen geworden. Ihre Prävalenz wird mit Raten von $30 \%$ bei Postmortem-Spenden und $16-20 \%$ bei Lebendleberspenden angegeben $[9,10]$. Die Lebersteatose wird in drei Grade eingeteilt: mild ( $<30 \%$ Steatose), moderat ( $\geq 30-60 \%$ Steatose) und schwer ( $\geq 60 \%$ Steatose; $[11,12])$, wobei eine Steatose $>40 \%$ ein Merkmal der ECD ist [2]. Die nichtalkoholische Fettleatmung $>7$ Tage, 3) Body-Mass-In$\operatorname{dex}(\mathrm{BMI})>30 \mathrm{~kg} / \mathrm{m}^{2}$, 4) Lebersteatose $>40 \%$, 5) Serumnatrium $>165 \mathrm{mmol} / \mathrm{l}$, 6) erhöhte Transaminasen (Alanin-Aminotransferase $[\mathrm{ALT}]>1750 \mathrm{nmol} /(\mathrm{s} \mathrm{l})$ [>105 U/l], Aspartat-Aminotransferase [AST] $>1500 \mathrm{nmol} /(\mathrm{sl})[>90 \mathrm{U} / \mathrm{l}]), 7) \mathrm{Se}-$ rumbilirubin $>51.3 \mu \mathrm{mol} / \mathrm{l} \quad[>3 \mathrm{mg} / \mathrm{dl}]$ [1]. Innerhalb der Eurotransplant-Region beträgt der Anteil an Lebertrans- 
- Spenderorgan transplantierbar?

- ja/nein

- unter Vorbehalt der makroskopischen Bewertung

- unter Vorbehalt der Leberbiopsie

- Allokation des Spenderorgans

- Alter des Empfängers

o an jedes Alter (auch Kinder)

o nur an Erwachsene

- alle Erwachsenen

- nur Erwachsene > 50 Jahre

- MELD-Score des Empfängers
o jeder MELD
o nur MELD $<30$
o nur MELD > 30

Abb. $1<$ Expertenevaluation von Spenderorgan und Allokation auf jeder Stufe der Umfrage. $M E L D$ "model of end stage liver disease" berkrankheit („non-alcoholic fatty liver disease", NAFLD) ist wiederum die Vorstufe der nichtalkoholischen Steatohepatitis (NASH), die bei Personen mit einem BMI $\geq 30-39 \mathrm{~kg} / \mathrm{m}^{2}$ in bis zu $20 \%$ und bei Personen mit einem BMI $<30 \mathrm{~kg} / \mathrm{m}^{2}$ in etwa $3 \%$ der Fälle nachgewiesen werden kann [12]. Transplantationen von Spenderorganen mit milder Steatose weisen ähnliche 5-Jahres-Überlebensraten wie nichtsteatotische Lebertransplantate auf [13]. Lebertransplantate mit moderatem Steatosegrad $(\geq 30-60 \%)$ sollten nur bei ausgewählten Empfängern [13-16] und Spenderorgane mit schwerer Steatose ( $\geq 60 \%$ Steatose) sollten nicht transplantiert werden $[1,17]$. Die makrovesikuläre Steatose ist ein unabhängiger Risikofaktor für das Transplantatüberleben, zusätzlich zu den im DRI beschriebenen Merkmalen [18]. Bemerkenswert in diesem Zusammenhang ist, dass ein deutsches Transplantationszentrum eine Zunahme stornierter Lebertransplantationen von $14,7 \% \mathrm{im} \mathrm{Jahr} 2010$ auf 63,6\% im Jahr 2016 aufgrund höhergradiger Lebersteatose berichtete [19].

Eine Laboruntersuchung vor Organentnahme ist zuverlässig zur Erkennung von Viruserkrankungen, aber weniger zuverlässig bei der Diagnose einer FLD. Die Serum-Gamma-Glutamyltransferase (GGT) ist ein empfindlicher Marker für die FLD, und erhöhte GGT-Spiegel sind mit einer fortgeschrittenen Fibrose bei der FLD assoziiert [20]. Die GGT ist jedoch als ein unspezifischer Marker anzusehen [21]. Der „FLD-Score“ (Alter, Hyperglykämie, BMI, Thrombozytenzahl, Albumin, AST/ALT-Verhältnis) erwies sich als zuverlässiges Instrument zur Vorhersage einer Fibrose bei FLD-Patienten [22]. Allerdings ist eine Steatose nicht unbedingt mit erhöhten Lebertransaminasen assoziiert [23], die in Bezug auf eine FLD eine niedrige Sensitivität aufweisen [24]. Zudem korrelieren biochemische Marker nicht mit dem Grad der Steatose [25].

In der klinischen Praxis wird die Ultraschalluntersuchung zur Detektion einer Lebersteatose (mild, moderat und schwer) eingesetzt. Die Ergebnisse und Reproduzierbarkeit der Sonographie unterliegen aber durchaus untersucherabhängigen Unterschieden [26]. Die histologische Untersuchung stellt daher immer noch den Goldstandard bei der Diagnose von Lebererkrankungen wie FLD, Fibrose und Entzündungen, aber auch bei der Graduierung von Lebersteatosen dar [1, 27-29]. Jedoch gehört die Leberbiopsie mit histologischer Befundung in der EurotransplantRegion nicht zur Standarddiagnostik zur Beurteilung von Spenderorganen [30]. Proben für eine histologische Untersuchung können entweder im Rahmen einer PLB („percutaneous liver biopsy“) vor oder als offene Keilbiopsie während der Organentnahme gewonnen werden. Für die Leberbiopsie während der Organentnahme mit histologischer Beurteilung werden in der Literatur Raten von $23-28 \%$ angegeben $[18,31]$. Für die PLB vor Organentnahme ergab eine kürzlich veröffentlichte Umfrage unter Teilnahme von 58 verschiedenen Organbeschaffungsorganisationen („organ procurement organizations", OPOs) Raten von nur 5-10\% [32]. Die Autoren kamen zu dem Schluss, dass „zusätzliche Informationen über den Nutzen, die Genauigkeit und die Sicherheit der PLB benötigt werden, um ihren Einsatz zu optimieren“.

Zusammenfassend sind der Stellenwert und die Sicherheit der PLB, ihre Auswirkungen auf die Organallokation, das Transplantationsergebnis und die Kosten bei der Lebertransplantation weiterhin unklar. Ziel unserer Studie war es daher, die Sicherheit der perkutanen PLB $\mathrm{zu}$ evaluieren, ihren Einfluss auf die Allokation von Lebertransplantaten hirntoter Spender zu analysieren und mögliche Auswirkungen auf wirtschaftliche und logistische Aspekte bei der Lebertransplantation zu veranschaulichen.

\section{Material und Methoden}

\section{Studiendesign}

Es handelt sich um eine retrospektive Beobachtungsstudie. Bei 36 hirntoten Spendern wurde eine PLB auf der Intensivstation vor geplanter Organentnahme durchgeführt.

\section{Spenderevaluation und Datenerhebung}

Alle ultraschallgesteuerten PLB wurden von einem in der Sonographie erfahrenen (DEGUM-Zertifikat) Transplantationschirurgen mit einer 18-Gauge-TruCut-Nadel durchgeführt, entsprechend unserer zuvor berichteten Technik [33]. Die Thrombozytenzahl war in allen Fällen $>50 / \mathrm{nl}$ und der Quick-Wert $>50 \%$. Die Gefrierschnitte wurden mit Hämatoxylin- und Eosinfärbung für die histologische Analyse durch einen erfahrenen Pathologen vorbereitet. Die Proben wurden auf Steatose $(<30 \%=$ mild, $30-40 \%=$ moderat, $>40 \%=$ schwer; makrovesikulär vs. mikrovesikulär), Fibro- 
se, Cholestase, Entzündung, Nekrose und andere Leberpathologien untersucht.

Durch die Deutsche Stiftung Organtransplantation (DSO) erfolgte nach der Feststellung des Hirntodes die Spenderevaluation. Diese bestand aus der Anamnese, den Basisspenderinformationen (Alter, Geschlecht, BMI), Laboruntersuchungen mit Virenscreening und einer Ultraschalluntersuchung der potenziellen Spenderleber. Die Sonographien wurden durch einen erfahrenen Transplantationschirurgen/Intensivmediziner durchgeführt. Ein 5-MHz-Konvexschallkopf wurde zur Ultraschallbeurteilung der Lebersteatose verwendet („KidneyTest“), die als „mild“ (erhöhte Echogenität der Leber gegenüber dem Nierenkortex), „moderat“ (Kriterien mild plus Unschärfe der intrahepatischen Gefäße) oder „schwer“ (Kriterien moderatplus abgeschwächte Darstellung der posterioren Lebersegmente und des Zwerchfells, „white liver“) graduiert wurde [26].

Anschließend wurde das Spenderorgan bei der Entnahme durch den Transplantationschirurgen makroskopisch beurteilt. Die Qualität des Spenderorgans wurde als "gut", „akzeptabel“ oder „schlecht/fett“ eingestuft.

Alle Daten wurden aus den Spenderberichten und medizinischen Aufzeichnungen der 36 hirntoten Spender entnommen.

\section{Umfragedesign}

Es wurde eine Umfrage unter Teilnahme von 11 Transplantationsexperten durchgeführt, um den Bedarf einer PLB und ihre Auswirkungen auf die Allokation zu bewerten. Hieraus ergaben sich 396 virtuelle Fälle (11 Experten $\times 36$ hirntote Spender). Die Umfrage bestand aus drei Stufen. Mit jeder Stufe erhielten die Experten zusätzliche Informationen zum Spender:

- Stufe 1: Anamnese, Basisinformationen zum Spender, Laborergebnisse, Ergebnis der Ultraschalluntersuchung;

- Stufe 2: Ergebnis der makroskopischen Einschätzung des Spenderorgans;

- Stufe 3: histologisches Ergebnis der Leberbiopsie (•Tab. 1).

Chirurg 2021 · 92:49-61 https://doi.org/10.1007/s00104-020-01192-w

(c) Der/die Autor(en) 2020

C. Beltzer · M. Quante - M. Rheinberger - H. A. Baba - F. Saner · F. Fend - T. Biet · A. Königsrainer $\cdot S$. Nadalin

\section{Perkutane Leberbiopsie vor Organentnahme - Einfluss auf Organallokation und Kosten in der Lebertransplantation}

\section{Zusammenfassung}

Hintergrund. Der Stellenwert und die Sicherheit einer perkutanen Leberbiopsie (PLB) bei hirntoten Spendern vor Organentnahme sowie der Einfluss der PLB auf die Organallokation und die Kosten im Rahmen der Lebertransplantation (LT) in der Eurotransplant-Region (ET), werden weiterhin diskutiert.

Material und Methoden. Eine perkutane Leberbiopsie vor Organentnahme erfolgte bei 36 hirntoten Spendern. Die Komplikationsrate, Spendercharakteristika, Ultraschallbefunde, die makroskopische Einschätzung und die histologischen Ergebnisse der PLB wurden analysiert. Zusätzlich wurde eine landesweite Umfrage unter 11 Lebertransplantationsexperten durchgeführt. Der Bedarf einer PLB und ihre Auswirkungen auf den Prozess der Organallokation wurden evaluiert. Mögliche Kosteneinsparungen wurden für verschiedene Szenarien auf der Grundlage von Kostendaten der Deutschen Stiftung Organtransplantation berechnet.
Ergebnisse. Es wurden keine Komplikationen durch die PLB beobachtet. Die Umfrage ergab, dass das Ergebnis der PLB einen erheblichen Einfluss auf die Allokation von Spenderorganen hat, insbesondere bei solchen mit "extended donor criteria (EDC)". Die Kostenberechnung ergab ein enormes Kosteneinsparungspotenzial durch eine optimierte Allokation und die Vermeidung unnötiger Organentnahmen.

Schlussfolgerung. Die PLB ist ein sicheres Verfahren und besitzt ein enormes Potenzial für die Optimierung der Organallokation vor Organentnahme durch eine Reduzierung der kalten Ischämiezeit, Vermeidung unnötiger Verwerfungen von Spenderorganen sowie Kosteneinsparungen. Die klinische Relevanz und der Einfluss der PLB auf die Organallokation konnte durch unsere Daten herausgestellt werden.

Schlüsselwörter

Kalte Ischämiezeit · Lebersteatose · Erweiterte Spenderkriterien · Spenderevaluation

\section{Percutaneous liver biopsy before organ removal-Impact on organ allocation and costs in liver transplantation}

\section{Abstract}

Background. The value and safety of percutaneous liver biopsy (PLB) in braindead donors before organ removal and its impact on organ allocation and costs of liver transplantation (LT) in the Eurotransplant (ET) region is still a matter of ongoing debate.

Material and methods. A PLB was performed in 36 brain-dead organ donors. The complication rate, ultrasonography findings, macroscopic evaluation and histological results of PLB and donor characteristics were analyzed. Additionally, a nationwide survey was conducted among 11 liver transplantation experts. The need for PLB and its impact on the liver allocation process were evaluated. Possible cost savings were calculated for different scenarios based on cost data provided by the German Organ Transplantation Foundation.

Results. No complications of PLB were observed. The survey revealed that the PLB has a substantial impact on the allocation of donor organs, especially in organs fulfilling extended donor criteria (EDC). The cost calculation revealed an enormous potential for cost savings due to an optimized organ allocation process and avoidance of futile organ procurement.

Conclusion. The PLB is a safe procedure and has tremendous potential for the optimization of the organ allocation process before organ procurement by reducing the cold ischemia time, avoiding unnecessarily discarding donor organs and saving costs. These data emphasize the clinical relevance and impact of PLB on the organ allocation process.

Keywords

Cold ischemia time - Liver steatosis · Extended donor criteria - Donor evaluation 


\section{Originalien}

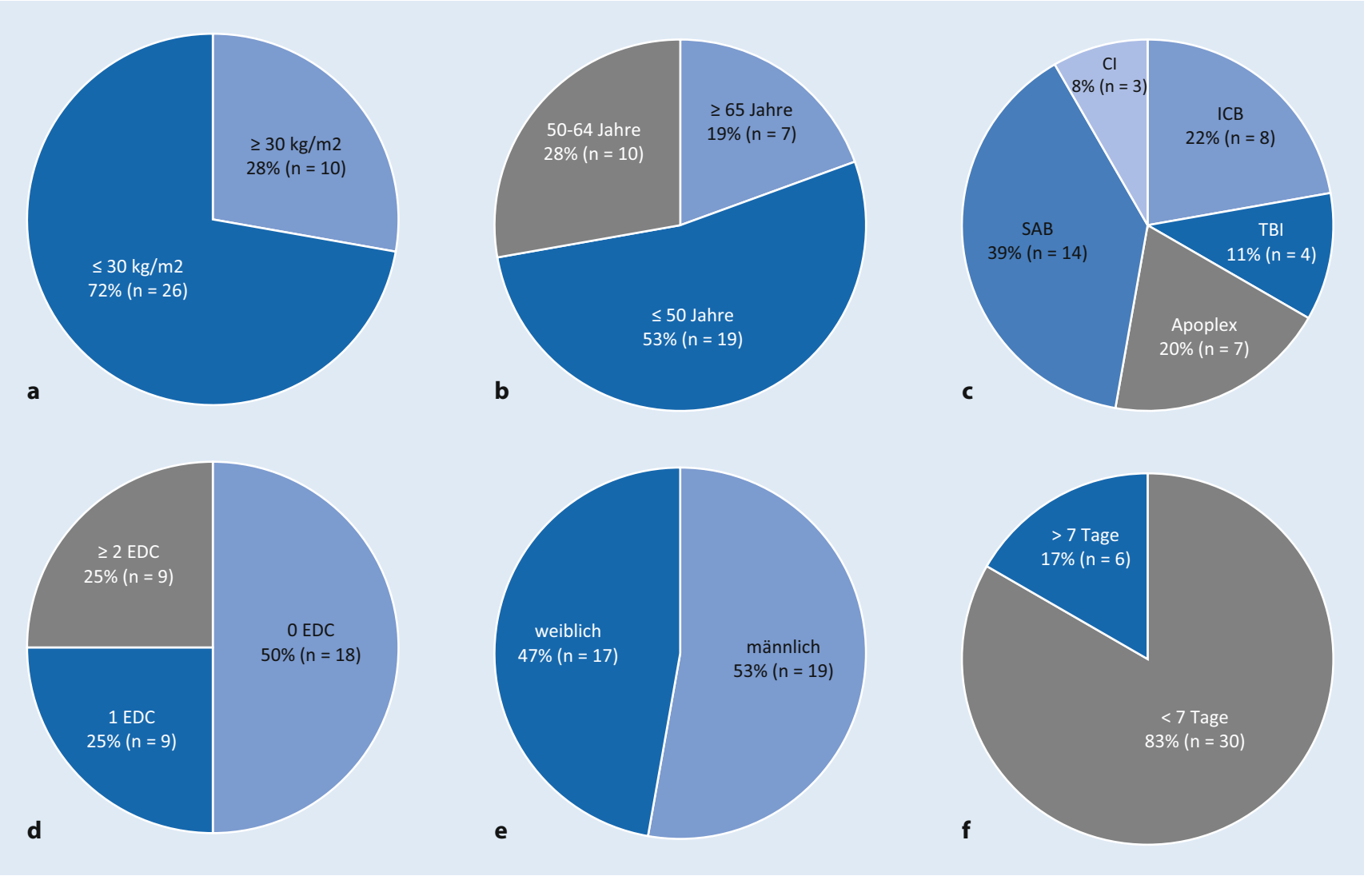

Abb. $2 \Delta$ Spendermerkmale. a Body-Mass-Index (BMI) . b Alter. c Ursachen des Hirntodes. d "Extended donor criteria“ (EDC), e Geschlecht. f Dauer des Intensivstations $(I C U)$-Aufenthaltes. Cl zerebrale Ischämie, ICB intrakranielle Blutung, SAB Subarachnoidalblutung, $T B I$ "traumatic brain injury"

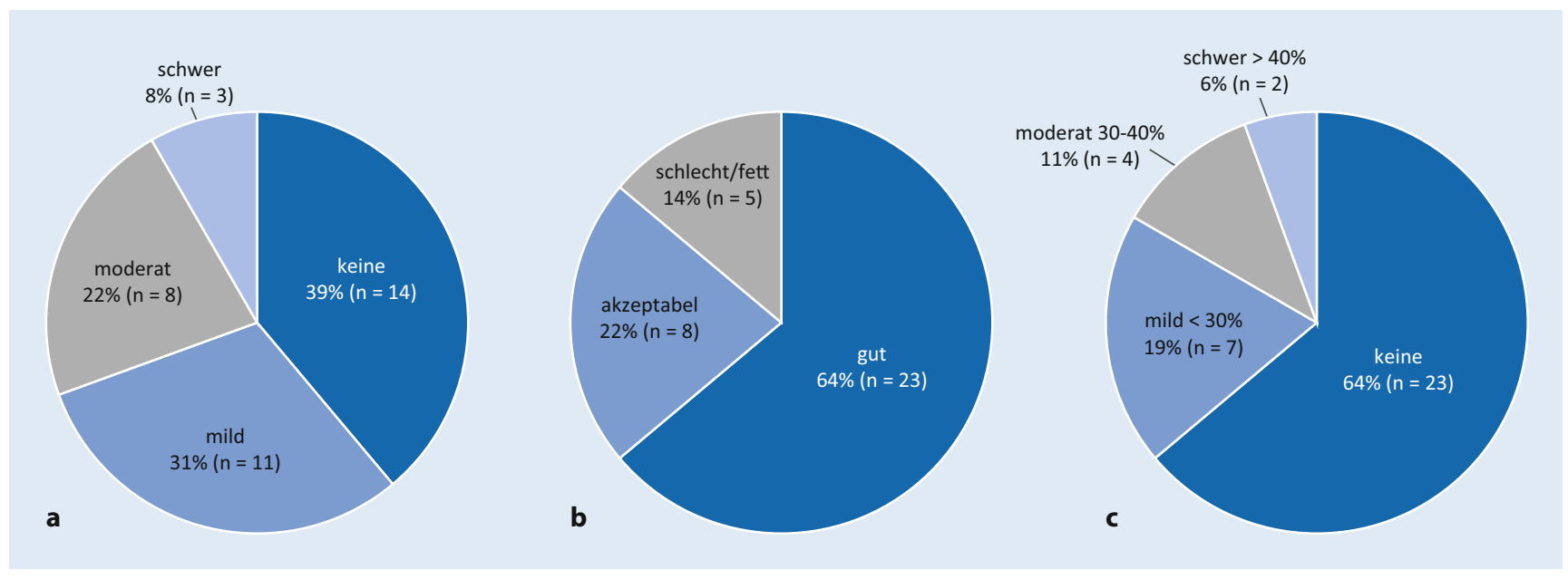

Abb. $3 \Delta$ a Steatose im Ultraschall, $\mathbf{b}$ in der makroskopischen Bewertung und c in der Leberbiopsie

Bei jeder Stufe mussten die Transplantationsexperten bewerten, ob das Spenderorgan transplantierbar ist und für welche Empfänger das Transplantat geeignet wäre (Alter: alle Altersgruppen einschließlich Kinder, nur Erwachsene, alle Erwachsenen, nur Alter >50 Jahre; MELD: alle MELD, nur MELD >30 oder nur MELD <30, • Abb. 1) oder ob sie weitere Informationen benötigen würden, um ihre Entscheidung zu treffen.

\section{Kostenberechnung}

Relevante Kostenfaktoren der Lebertransplantation (LT) wurden von der Deutschen Stiftung Organstransplantation (DSO) bezogen und auf der 
Tab. 2 Graduierung der Lebersteatose mittels Ultraschall, makroskopischer Bewertung und Leberbiopsie

\begin{tabular}{|c|c|c|c|}
\hline & $\begin{array}{l}\text { Gut (keine } \\
\text { Steatose oder } \\
<30 \% \text { : mild) }\end{array}$ & $\begin{array}{l}\text { Akzeptabel (Steatose } \\
\text { 30-40\%: moderat) }\end{array}$ & $\begin{array}{l}\text { Schlecht (Steatose } \\
>40 \% \text { : schwer) }\end{array}$ \\
\hline Ultraschall & $25(69 \%)$ & $8(22 \%)$ & $3(8 \%)$ \\
\hline $\begin{array}{l}\text { Makroskopische Bewer- } \\
\text { tung }\end{array}$ & 23 (64\%) & $8(22 \%)$ & $5(14 \%)$ \\
\hline Leberbiopsie & 30 (83\%) & $4(11 \%)$ & $2(6 \%)$ \\
\hline
\end{tabular}

Tab. 3 Befunde der Leberbiopsie: alle Spender, Spender mit BMl $<30 \mathrm{~kg} / \mathrm{m}^{2}$ und Spender mit $\mathrm{BMI} \geq 30 \mathrm{~kg} / \mathrm{m}^{2}$. Spender können in mehr als einer Kategorie vorkommen

\begin{tabular}{|c|c|c|c|}
\hline Pathologie & $\begin{array}{l}\text { Alle Spender } \\
(n=36)\end{array}$ & $\begin{array}{l}\text { Spender BMI } \\
<30 \mathrm{~kg} / \mathrm{m}^{2} \\
(n=26)\end{array}$ & $\begin{array}{l}\text { Spender BMI } \\
\geq 30 \mathrm{~kg} / \mathrm{m}^{2} \\
(n=10)\end{array}$ \\
\hline \multicolumn{4}{|l|}{ Steatotisch;n (\%) } \\
\hline Makrosteatose $40 \%$ & $2(5,5)$ & $1(3,8)$ & $1(10,0)$ \\
\hline Makrosteatose $30 \%$ & $4(11,1)$ & $3(11,5)$ & $1(10,0)$ \\
\hline Makrosteatose $<20 \%$ & $7(11,1)$ & $4(15,4)$ & $3(30,0)$ \\
\hline Mikrosteatose & $13(30,5)$ & $8(30,8)$ & $5(50,0)$ \\
\hline Gemischte Steatose & $9(22,2)$ & $4(15,4)$ & $5(50,0)$ \\
\hline \multicolumn{4}{|l|}{ Nichtsteatotisch;n (\%) } \\
\hline Vakuolardegeneration & $1(2,8)$ & $1(3,8)$ & - \\
\hline $\begin{array}{l}\text { Unspezifischer Hepatozy- } \\
\text { tenschaden }\end{array}$ & $1(2,8)$ & $1(3,8)$ & - \\
\hline Leichtgradige Fibrose & $2(2,8)$ & $1(3,8)$ & $1(10,0)$ \\
\hline Zellschwellung & $1(2,8)$ & $1(3,8)$ & - \\
\hline Cholestase & $6(16,6)$ & $2(7,6)$ & $4(40,0)$ \\
\hline Periportale Entzündung & $1(2,8)$ & - & $1(10,0)$ \\
\hline
\end{tabular}

Tab. 4 Korrelation der Steatose zwischen Ultraschall und Leberbiopsie

\begin{tabular}{|c|c|c|c|}
\hline & $\begin{array}{l}\text { Leberbiopsie: „moderat“ } \\
\text { oder "schwer" }\end{array}$ & $\begin{array}{l}\text { Leberbiopsie: „keine“ } \\
\text { oder „mild“ }\end{array}$ & Total \\
\hline $\begin{array}{l}\text { Ultraschall: „,moderat" } \\
\text { oder „schwer" }\end{array}$ & $\begin{array}{l}5 \\
\text { (richtig-positiv) }\end{array}$ & $\begin{array}{l}6 \\
\text { (falsch-positiv) }\end{array}$ & 11 \\
\hline $\begin{array}{l}\text { Ultraschall: „keine“ oder } \\
\text { „mild“ }\end{array}$ & $\begin{array}{l}1 \\
\text { (falsch-negativ) }\end{array}$ & $\begin{array}{l}24 \\
\text { (richtig-negativ) }\end{array}$ & 25 \\
\hline Total & 6 & 30 & 36 \\
\hline
\end{tabular}

Tab. 5 Korrelation der Steatose zwischen makroskopischer Bewertung und Leberbiopsie

\begin{tabular}{|c|c|c|c|}
\hline & $\begin{array}{l}\text { Leberbiopsie: „moderat“ } \\
\text { oder "schwer“" }\end{array}$ & $\begin{array}{l}\text { Leberbiopsie: „keine“ } \\
\text { oder „mild“ }\end{array}$ & Total \\
\hline $\begin{array}{l}\text { Makroskopische Bewer- } \\
\text { tung: „moderat" oder } \\
\text { "schwer" }\end{array}$ & $\begin{array}{l}6 \\
\text { (richtig-positiv) }\end{array}$ & $\begin{array}{l}7 \\
\text { (falsch-positiv) }\end{array}$ & 13 \\
\hline $\begin{array}{l}\text { Makroskopische Bewer- } \\
\text { tung: „keine“ oder „mild“ }\end{array}$ & $\begin{array}{l}0 \\
\text { (falsch-negativ) }\end{array}$ & $\begin{array}{l}23 \\
\text { (richtig-negativ) }\end{array}$ & 23 \\
\hline Total & 6 & 30 & 36 \\
\hline
\end{tabular}

Grundlage der bereitgestellten Daten berechnet.

\section{Auswertung und statistische Analyse}

Die Auswertung der Umfrage bestand aus a) der Häufigkeit der Anforderung weiterer Spenderinformationen; b) dem Einfluss der Spenderinformationen auf die Allokation und auf die Auswahl geeigneter Empfänger; c) der Bewertung der Spendermerkmale, die die Entscheidungsfindung beeinflussen.

Zum Vergleich kategorialer Variablen wurde der exakte Fisher-Test verwendet, zum Vergleich normalverteilter Variablen wurde ein ungepaarter $\mathrm{t}$-Test mit der Welch-Korrektur verwendet. Die Ergebnisse werden in Mittelwert \pm Standardabweichung oder Median und Interquartilsbereich angegeben. $p$-Werte $<0,05$ wurden als signifikant angesehen. Die Statistiken wurden mit Microsoft Excel 2010 (Microsoft Corporation, Washington) und GraphPad Prism 4 (GraphPad Software, Inc.) berechnet.

\section{Ergebnisse}

\section{Komplikationen der PLB}

Es wurden keine Komplikationen im $\mathrm{Zu}$ sammenhang mit der PLB beobachtet, d.h. keine intra- oder extrahepatischen Hämatome, keine Galleleckagen oder andere Verletzungen, die die Spenderleber beeinträchtigten. Es kam zu keinem Transplantatverlust durch die PLB.

\section{Merkmale der Spender,}

makroskopische Bewertung, Ultraschallergebnisse, Histologie, Korrelation der diagnostischen Tests

\section{Merkmale der Spender und Vorliegen erweiterter Spenderkriterien (० Abb. 2)}

Die Todesursachen der 36 hirntoten Spender waren: subarachnoidale Blutung bei 14 Spendern (39\%), intrakranielle Blutungen bei 8 (22\%), Apoplex bei 7, traumatische Hirnverletzung bei 4 (11\%) und zerebrale Ischämie bei 3 (8\%). 


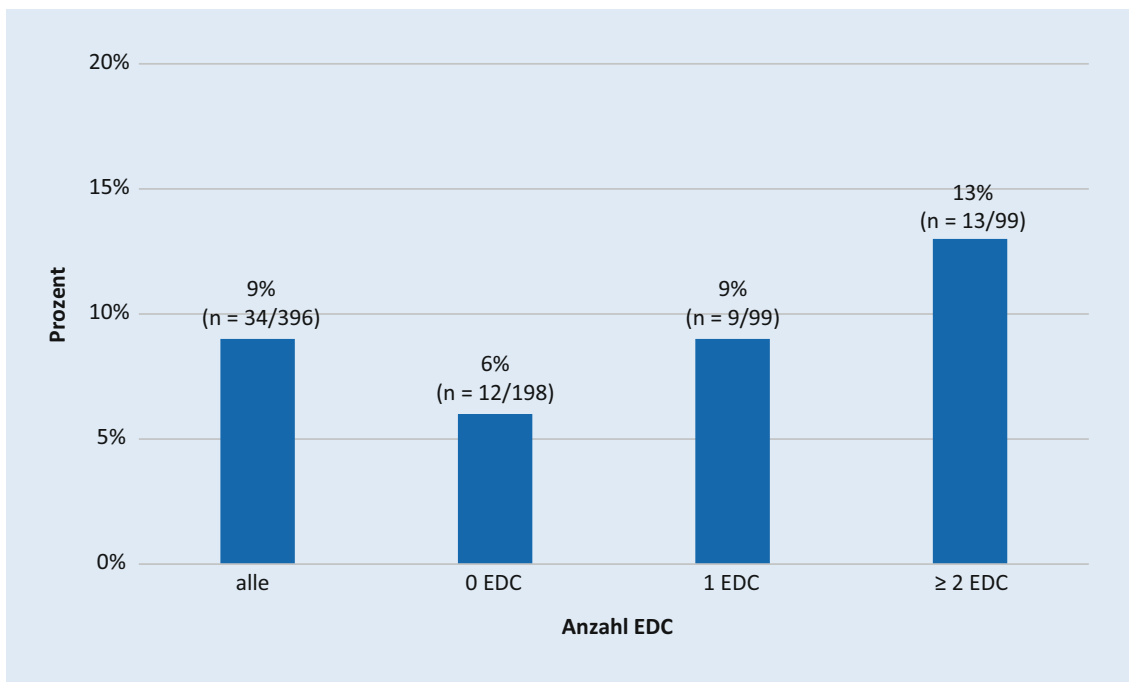

Abb. $4 \Delta$ Nachfrage nach makroskopischer Bewertung in Abhängigkeit von der Anzahl vorliegender „extended donor criteria“ (EDC)

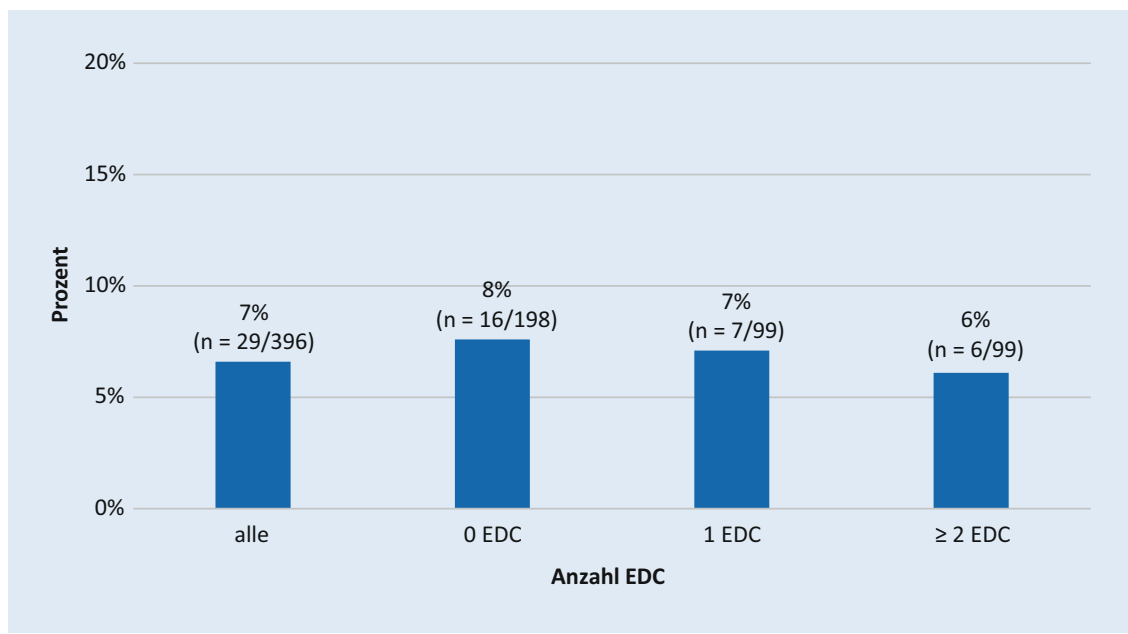

Abb. 5 \ Änderung der Allokation nach makroskopischer Bewertung in Abhängigkeit von der Anzahl vorliegender "extended donor criteria" (EDC)

Das mittlere Spenderalter betrug 49,2 $\pm 17,4$ Jahre, wobei 19 Spender $<50$ Jahre (52\%), 10 Spender 50 bis 64 Jahre $(28 \%)$ und $7>65$ Jahre (19\%) alt waren. 19 waren männlich (53\%) und 17 weiblich (47\%). Der durchschnittliche BMI betrug $27,2 \pm 4,7 \mathrm{~kg} / \mathrm{m}^{2}$, mit 26 Spendern $<30 \mathrm{~kg} / \mathrm{m}^{2} \quad(72 \%)$ und 10 Spendern $>30 \mathrm{~kg} / \mathrm{m}^{2}$ (28\%). Die durchschnittliche Aufenthaltsdauer auf der Intensivstation betrug 5,3 $\pm 4,8$ Tage. 30 Spender hatten einen Intensivaufenthalt von $<7$ Tagen ( $83 \%$ ) und 6 Spender $>7$ Tage (17\%). Die Laborergebnisse der Spender zeigten eine mittlere Serum-Aspartat-Aminotransferase (AST) von $480 \mathrm{nmol} /(\mathrm{s} \mathrm{l}) \pm 326 \mathrm{nmol} /(\mathrm{s} \mathrm{l})$ oh- als "moderate Steatose“ und $3(8 \%)$ als „schwere Steatose“ (• Abb. 3; • Tab. 2).

\section{Makroskopische Bewertung während der Organentnahme}

Bei der makroskopischen Beurteilung der Spenderlebern durch den Transplantationschirurgen während der Organentnahme wurden $23(64 \%)$ als "gut", $8(22 \%)$ als "akzeptabel“ und $5(14 \%)$ als „schlecht/fett" bewertet (ब Abb. 3; - Tab. 2).

\section{Histologische Ergebnisse der PLB}

Die histologische Untersuchung der Leberbiopsien ergab bei 21 der 36 Spender (58\%) den Befund einer Steatose, davon bei 13 (36\%) eine Makrosteatose (- Tab. 3). Die Makrosteatose wurde bei 2 Spendern $(6 \%)$ als „schwer" (Steatose $>40 \%)$, bei $4(11 \%)$ als "moderat" (Steatose $30-40 \%)$ und bei 7 (19\%) als "mild“ (Steatose <30\%) eingestuft (- Abb. 3; - Tab. 2). Eine Makrosteatose $>30 \%$ war bei Spendern mit BMI $\geq 30 \mathrm{~kg} / \mathrm{m}^{2}$ häufiger als bei Spendern mit BMI $<30 \mathrm{~kg} / \mathrm{m}^{2}$, aber zwischen den BMI-Gruppen statistisch nicht signifikant $(p=0,7)$.

Andere, nichtsteatotische Pathologien (leichtgradige Fibrose, Cholestase, unspezifischer Hepatozytenschaden, Vakuolardegeneration, periportale Entzündung, Zellschwellung) wurden bei 10 Spendern (28\%) diagnostiziert (•Tab.3).

\section{Korrelation von Ultraschall und Histologie}

Sonographisch wurden 3 Spenderorgane als „schwere "Lebersteatose eingestuft. Histologisch zeigten davon 2 Leberbiopsien eine „schwere“ ( $40 \%$ Makrosteatose) und 1 eine „moderate "Steatose (30\% Makrosteatose). Von den 8 sonographisch als "moderat" steatotisch eingestuften Lebern wiesen 4 histologisch keine Steatose auf.

Insgesamt wurden sonographisch 25 Spenderorgane als "keine Steatose“ oder "mild“ steatotisch klassifiziert. Unter diesen lag histologisch nur in einem Fall eine „moderate“ Makrosteatose vor.

Hieraus resultierten eine Sensitivität und Spezifität des Ultraschalls zur Erkennung einer "moderaten“ und „schweren“ 


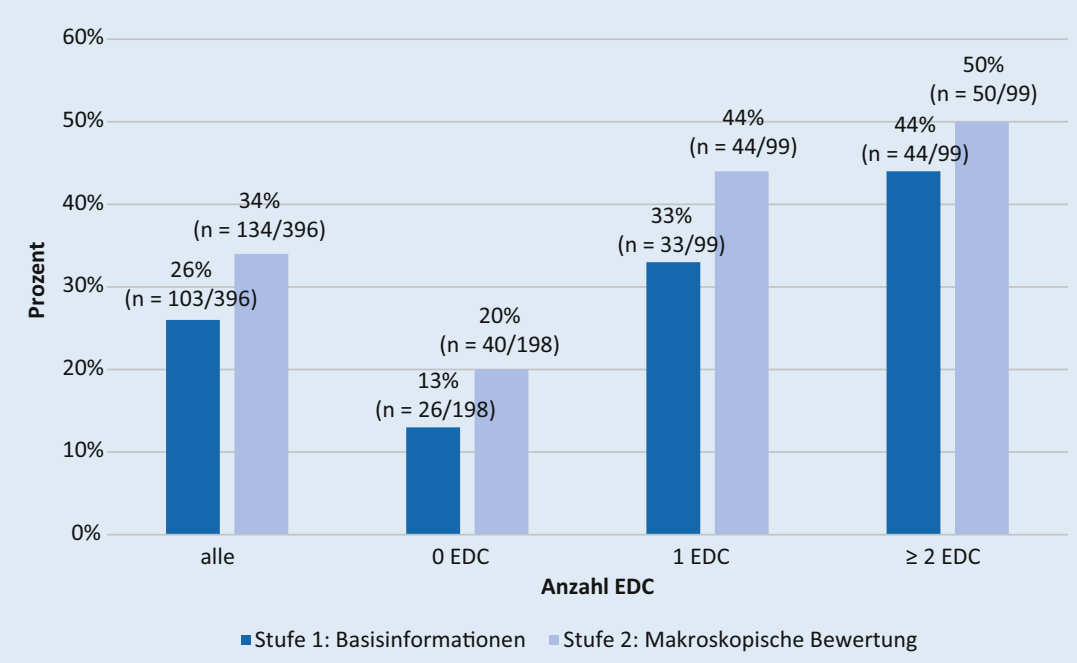

Abb. $6 \Delta$ Nachfrage nach einer Leberbiopsie auf Stufe 1 (Basisinformationen) und Stufe 2 (makroskopische Bewertung) in Abhängigkeit von der Anzahl an „extended donor criteria“ (EDC)

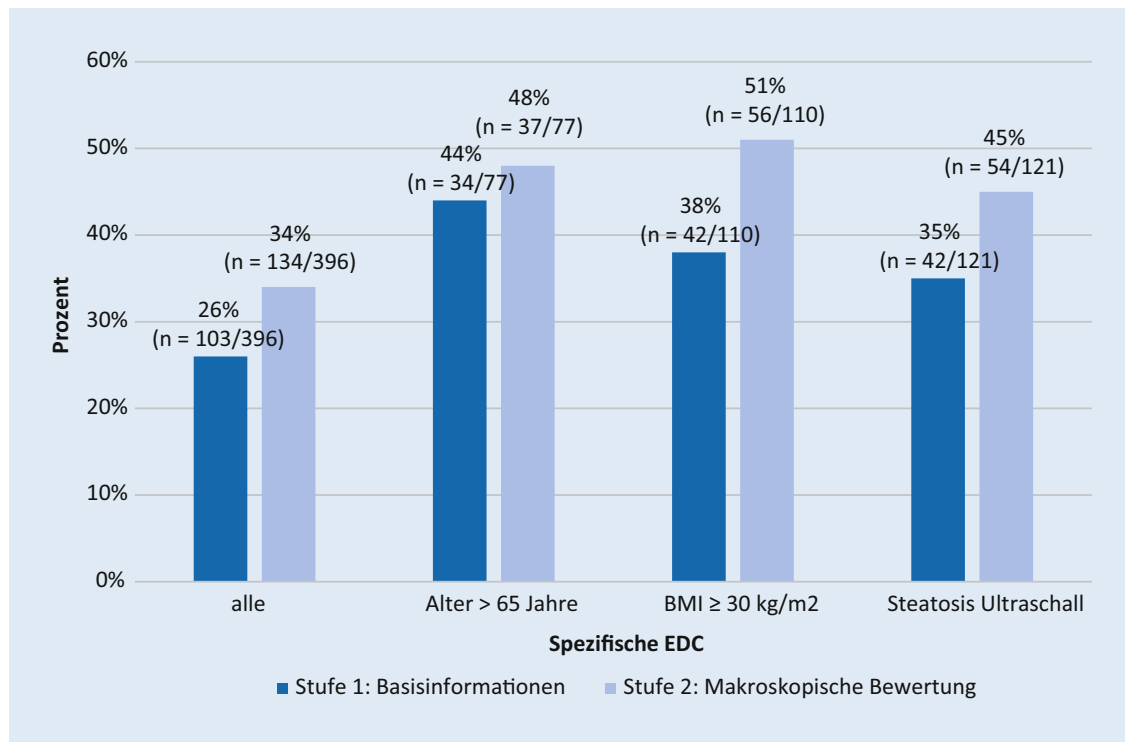

Abb. 7 ॥ Nachfrage nach einer Leberbiopsie bei verschiedenen „extended donor criteria“ (EDC) auf Stufe 1 (Basisinformationen) und Stufe 2 (makroskopische Bewertung). Spender können in mehr als einer Kategorie vorkommen. BMI Body-Mass-Index

Makrosteatose $\geq 30 \%$ von 83 bzw. $80 \%$ (• Tab. 4).

\section{Korrelation von makroskopischer Bewertung und Histologie}

Insgesamt 13 Spenderlebern wurden makroskopisch als "akzeptabel“ oder „schlecht/fett“ eingestuft. Unter diesen lag histologisch in 6 Fällen eine „moderate“ oder "schwere“ Makrosteatose $\geq 30 \%$ vor, 7 wiesen „keine“ oder eine „milde“ Makrosteatose auf.

\section{Umfrage}

\section{Bedarf einer makroskopischen Bewertung}

Eine makroskopische Bewertung wurde in $9 \%$ der Fälle gefordert. Die Nachfrage einer makroskopischen Bewertung stieg parallel zur Anzahl der vorliegenden EDC: $6 \%$ ohne EDC, $9 \%$ mit 1 EDC und $13 \%$ mit $\geq 2 \operatorname{EDC}$ (• Abb. 4).

\section{Änderungen der Allokation durch die makroskopische Bewertung}

Die makroskopische Bewertung führte in $7 \%$ aller Fälle zu einer Änderung der Allokation. Es gab keine signifikanten Unterschiede zwischen Spendern mit und ohne EDC (• Abb. 5). In $69 \%$ der Fälle, die zu einer Änderung der Allokation führten, verlangten die Experten zunächst keine makroskopische Bewertung.

\section{Bedarf einer Leberbiopsie mit Histologie}

Eine Leberbiopsie wurde in $26 \%$ der Fälle nach Stufe 1 (Basisinformationen zum Spender) verlangt. Die Nachfrage stieg nach Schritt 2 (makroskopische Bewertung) auf $34 \%$. Darüber hinaus stieg die Nachfrage nach einer Leberbiopsie signifikant mit der Anzahl der EDC an, mit einem Maximum von $50 \%$ bei Spendern mit $\geq 2$ EDC (• Abb. 6).

Eine Subgruppenanalyse 3 verschiedener EDC (Alter >65 Jahre; BMI $\geq 30 \mathrm{~kg} / \mathrm{m}^{2}$; Steatose bei Ultraschall) und die damit einhergehende spezifische Häufigkeit der Anforderung einer Leberbiopsie ist in $\bullet$ Abb. 7 dargestellt. Ein Alter $>65$ Jahre wurde als das EDC mit der höchsten Nachfrage nach einer Leberbiopsie nach Stufe 1 (44\%) identifiziert, ein $\mathrm{BMI} \geq 30 \mathrm{~kg} / \mathrm{m}^{2}$ ergab die höchste Nachfrage nach Leberbiopsie nach Schritt 2 (51\%).

\section{Änderung der Allokation durch die Leberbiopsie}

Eine Leberbiopsie führte in $19 \%$ aller Fälle zu einer Änderung der Allokation. Diese nahm mit der Anzahl der EDC zu: $14 \%$ Änderungen der Allokation bei Spendern ohneEDC, $23 \%$ mit 1 EDC und $26 \%$ mit $\geq 2$ EDC (• Abb. 8). In $50 \%$ der Fälle mit Änderung der Allokation nach 


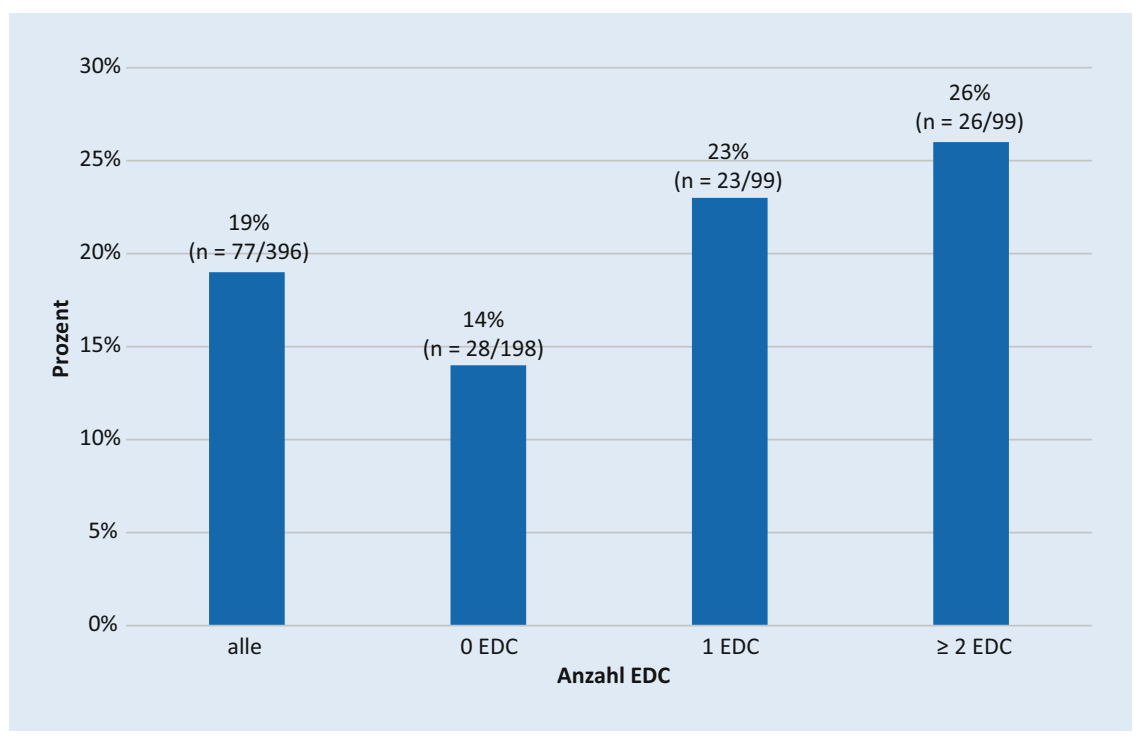

Abb. 8 ॥ Änderung der Allokation nach Leberbiopsie in Abhängigkeit von der Anzahl an „extended donor criteria" (EDC)

Leberbiopsie gaben die Experten initial an, diese nicht zu benötigen.

\section{Kostenberechnung}

Daten zur Kostenberechnung im $\mathrm{Zu}$ sammenhang mit der Organentnahme und der Allokation wurden von der Deutschen Stiftung Organtransplantation (DSO) zur Verfügung gestellt. Im Fall einer unnötigen Organentnahme mit anschließendem Transport, bei dem die Leber aufgrund des histologischen Ergebnisses letztendlich verworfen werden muss, ergeben sich Kosten (Personal, Logistik und Material) zwischen $4685 €$ (Bodentransport) und $10.895 €$ (Lufttransport). Im Falle einer bereits veranlassten stationären Aufnahme eines designierten Transplantatempfängers mit präoperativer Vorbereitung (Labordiagnostik, Röntgen, Vorbereitung der Operation und Transplantationsteam) fallen zusätzliche Kosten von mindestens $2500 €$ an.

Insgesamt 491 Lebertransplantate, die in Deutschland von 2010 bis 2016 alloziert wurden, wurden schließlich nicht transplantiert [34]. Dies ergibt durchschnittlich 70 unnötige Organentnahmen und Allokationen jährlich. Diese Zahl muss mit den oben genannten Kosten multipliziert werden, um das Einsparungspotenzial in Deutschland pro
Jahr durch eine PLB vor Organentnahme zu berechnen.

\section{Diskussion}

In Deutschland bestehen bei der Beurteilung der Organqualität von Spenderlebern weiterhin regelmäßig Diskrepanzen zwischen sonographischer und makroskopischer Einschätzung einerseits und dem histologischen Befund, welcher in der Regel erst vorliegt, nachdem die Allokation bereits gestartet wurde. Mögliche Folgen sind ein "mismatch" zwischen Lebertransplantat und Empfänger sowie eine nachträgliche Änderung der Allokation mit Verlängerung der kalten Ischämiezeit.

Ziel unserer Studie war es, die Sicherheit der PLB vor Organentnahme auf Intensivstation und ihre Auswirkungen auf Allokation und Kosten bei der LT zu bewerten.

Es traten keine Komplikationen durch die ultraschallgesteuerte PLB auf, womit diese sich als sicheres invasives diagnostisches Verfahren erwies, was durch die Berichte von Savas et al. [9], Nadalin et al. [33] sowie Ryan et al. [35] untermauert wird. In den zitierten Studien traten bei potenziellen Leberlebendspendern in 201, 144 und 100 durchgeführten PLB keine Komplikationen auf. Aktuelle Daten berichten über Komplikationsraten nach PLB um 0,57\%, und
Komplikationen steigen mit der Anzahl der Biopsieversuche [36]. Bezüglich der Mindestqualifikation des Biopsierenden sahen Westheim et al. eine erhöhtes postinterventionelles Blutungsrisiko bei vorliegender Erfahrung von <20 Biopsien $(3,2 \%)$ gegenüber $>100$ Biopsien $(1,1 \%$; [37]). Bei einem Durchmesser der Biopsienadel $>1,6 \mathrm{~mm}$ kann es ebenfalls zu vermehrten Blutungskomplikationen kommen [38]. Aus diesem Grund empfehlen wir folgende Standards für die PLB: ultraschallgesteuerte Biopsie, vorhandene Erfahrung des Durchführenden in Form von mindestens $>20$ Biopsien, nicht mehr als ein Biopsieversuch und Verwendung einer 18-Gauge-Tru-Cut-Nadel [39].

Unsere Ergebnisse zeigten eine nicht ausreichend zuverlässige Korrelation zwischen Ultraschallbefunden und makroskopischer Bewertung mit den Ergebnissen der Leberbiopsie in Bezug auf das Vorliegen sowie die Gradierung einer Steatose. Für die Leberbiopsie konnten wesentliche Vorteile zur Beurteilung von Spenderlebern demonstriert werden. Sowohl die Ultraschalluntersuchung als auch die makroskopische Bewertung stuften die Spenderlebern in 20 und 23\% der Fälle als "moderate“ oder „schwere“ Makrosteatose ein, während histologisch nur eine "geringe“ oder gar "fehlende" Makrosteatose vorlag. Andere Studien zeigten eine noch geringere Korrelation zwischen Ultraschall [9], makroskopischer Bewertung [40] und histologischen Ergebnissen. Dies kann dazu führen, dass Spenderorgane aufgrund der sonographischen oder makroskopischen Einschätzung fälschlicherweise verworfen werden, insbesondere da eine sonographisch diagnostizierte Lebersteatose als ein Hauptgrund für die Verwerfung von Spenderorganen gilt [41]. Czerwinski et al. zeigten, dass $35 \%$ der verworfenen Lebertransplantate bei der abschließenden histologischen Untersuchung nur minimale Veränderungen aufwiesen und somit zur Transplantation hätten verwendet werden können [42]. Die histologische Untersuchung ist nach wie vor der Goldstandard für den Nachweis einer Steatose und anderen Leberpathologien wie NASH, Fibrose, Entzündung, Cholestase oder unspezifischer Hepatitis [24], welche allesamt ebenfalls eine Kon- 


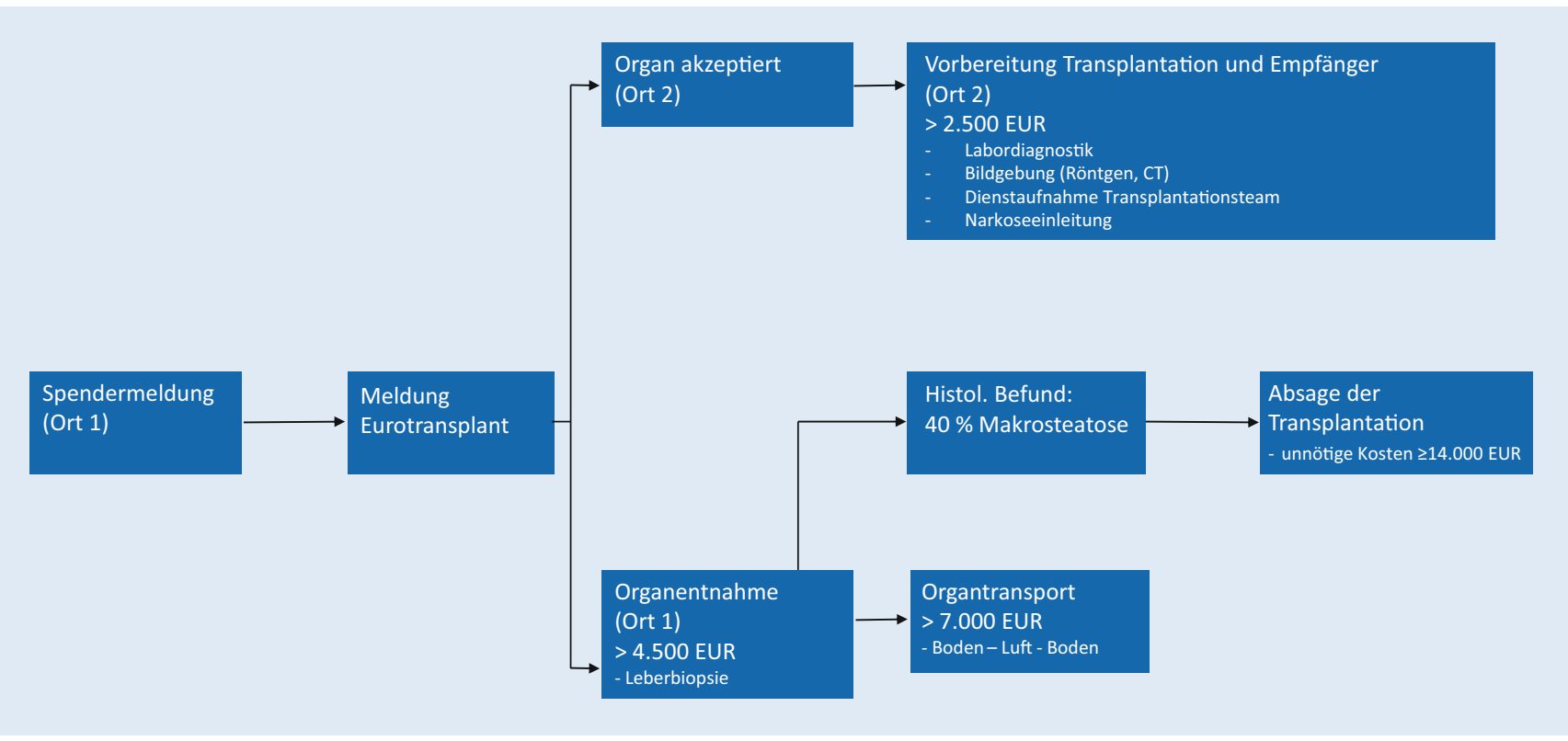

Abb. 9 ॥ Allokation und Kosten ohne „percutaneous liver biopsy“ (PLB) auf der Intensivstation. CT Computertomographie

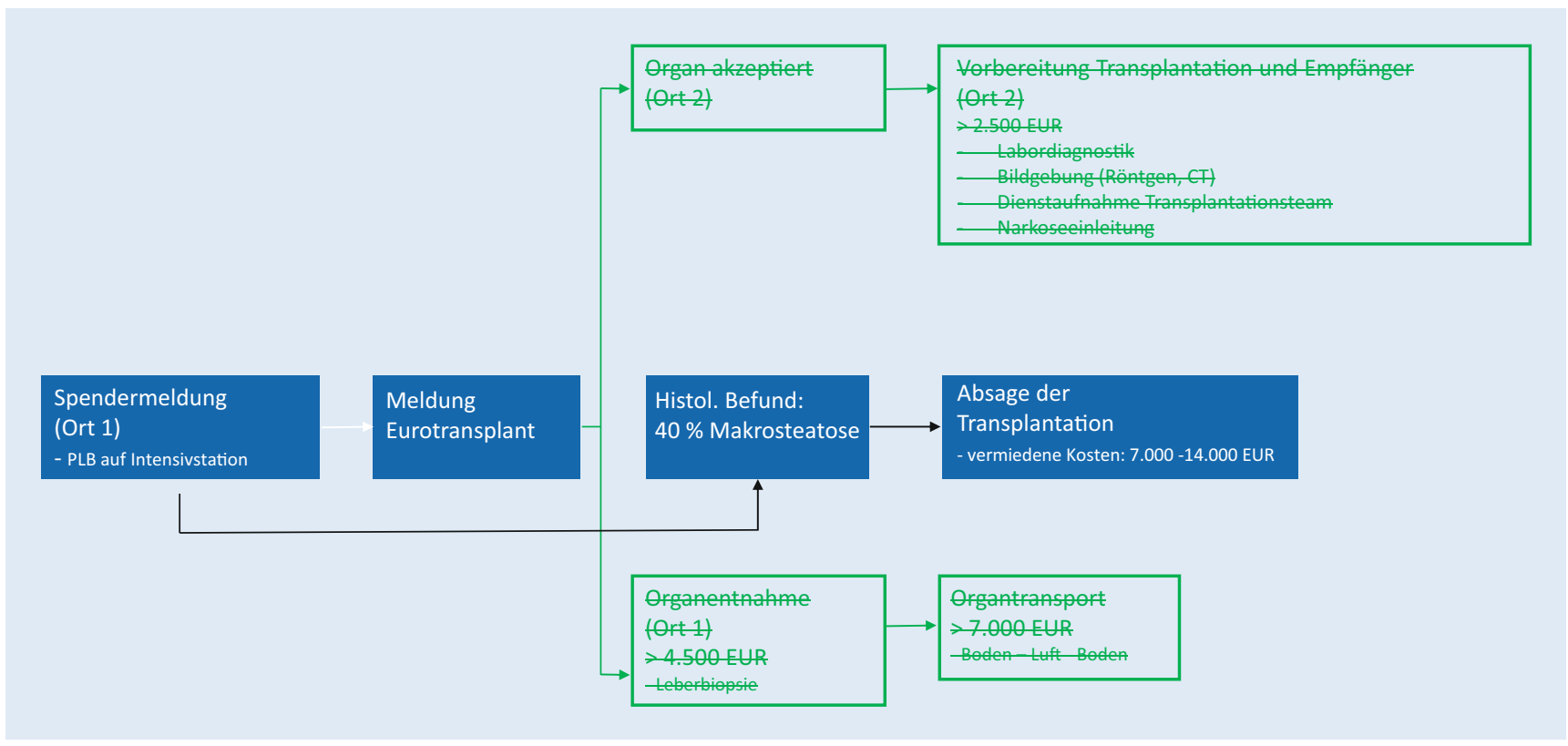

Abb. $10 \triangle$ Allokation und Kosten mit „percutaneous liver biopsy“ (PLB) auf der Intensivstation. Grün vermeidbare Kosten durch PLB

traindikation für eine Transplantation darstellen können.

Unsere Umfrage zeigte, dass die PLB eine entscheidende Rolle in der Allokation spielt, bei der sie in 19\% der Fälle zu einer Änderung führte. Bei Vorhandensein von EDC stieg der Einfluss der PLB auf die Allokation weiter an $(23 \%$ bei Spendern mit 1 EDC, $26 \%$ in Fällen mit $\geq 2$ EDC), was ihren Stellenwert insbesondere bei ECD-Spendern unterstreicht. In- teressanterweise verlangten die Experten in fast $50 \%$ der Fälle mit Änderung der Allokation nach Erhalt des histologischen Befundes zunächst keine Leberbiopsie.

Im Gegensatz dazu führte die makroskopische Bewertung nur in $7 \%$ aller Fälle zu einer Änderung der Allokation, ohne dass es einen signifikanten Unterschied zwischen der Anzahl an vorliegenden EDC gab.
Yersiz et al. [43] zeigten, dass eine makrovesikuläre Steatose vom Transplantationschirurgen anhand der Lebermorphologie (Gelbfärbung, abgerundete Kanten) mit einer Genauigkeit von bis zu $86 \%$ vorhergesagt werden kann. Für eine makroskopische Graduierung der Steatose sank die Genauigkeit jedoch auf nur $75 \%$. Unter anderem auf Grundlage dieser Ergebnisse haben die Deutsche Transplantationsgesellschaft, die Deut- 


\begin{tabular}{|c|c|}
\hline \multicolumn{2}{|c|}{ Abkürzungen } \\
\hline$A L T$ & Alanin-Aminotransferase \\
\hline AST & Aspartat-Aminotransferase \\
\hline$B A R$ & Balance of risk (score) \\
\hline$B D D$ & $\begin{array}{l}\text { Brain-dead donors, hirntote } \\
\text { Spender }\end{array}$ \\
\hline$B M I$ & Body-Mass-Index \\
\hline $\mathrm{Cl}$ & Zerebrale Ischämie \\
\hline CIT & $\begin{array}{l}\text { Cold ischemia time, kalte } \\
\text { Ischämiezeit }\end{array}$ \\
\hline$C T$ & Computertomographie \\
\hline DEGUM & $\begin{array}{l}\text { Deutsche Gesellschaft für } \\
\text { Ultraschall in der Medizin }\end{array}$ \\
\hline$D R I$ & Donor risk index \\
\hline DSO & $\begin{array}{l}\text { Deutsche Stiftung Organtrans- } \\
\text { plantation }\end{array}$ \\
\hline$E C D$ & $\begin{array}{l}\text { Extended criteria donors, Spender } \\
\text { mit erweiterten Spenderkriterien }\end{array}$ \\
\hline$E D C$ & $\begin{array}{l}\text { Extended donor criteria, erweiter- } \\
\text { te Spenderkriterien }\end{array}$ \\
\hline$E T$ & Eurotransplant \\
\hline$F L D$ & $\begin{array}{l}\text { Fatty liver disease, Fettleberer- } \\
\text { krankung }\end{array}$ \\
\hline GGT & Gamma-Glutamyltransferase \\
\hline$I C B$ & Intrakranielle Blutung \\
\hline$I C U$ & Intensive care unit, Intensivstation \\
\hline$L T$ & Lebertransplantation \\
\hline MELD & Model of end stage liver disease \\
\hline NASH & $\begin{array}{l}\text { Non-alcoholic steatohepatitis, } \\
\text { nichtalkoholische Hepatitis }\end{array}$ \\
\hline OLT & $\begin{array}{l}\text { Orthotopic liver transplantation, } \\
\text { orthotope Lebertransplantation }\end{array}$ \\
\hline OPO & Organ procurement organization \\
\hline$P L B$ & Percutaneous liver biopsy \\
\hline$P N F$ & Primary non function \\
\hline$S A B$ & Subarachnoidalblutung \\
\hline$T B I$ & $\begin{array}{l}\text { Traumatic brain injury, traumati- } \\
\text { scher Hirnschaden }\end{array}$ \\
\hline USD & US-Dollar \\
\hline
\end{tabular}

sche Stiftung Organtransplantation und die Deutsche Gesellschaft für Pathologie kürzlich Indikationen für die Gewinnung einer Leberbiopsie während der Organentnahme definiert (Schätzung der Lebersteatose durch den Transplantationschirurgen $\geq 30 \%$; [30]).
Eine PLB bietet im Vergleich zur offenen Biopsie während der Organentnahme mehrere Vorteile, mit entsprechend hohem Potenzial zur Verbesserung des Outcomes bei der Lebertransplantation. Das histologische Ergebnis der PLB liegt bereits vor geplanter Organentnahme vor. Hierdurch kann die Auswahl eines idealen Transplantatempfängers in einem frühen Stadium des Allokationsverfahrens erfolgen und eine Änderung der Allokation mit damit einhergehender Verlängerung der kalten Ischämiezeit vermieden werden. Eine lange kalte Ischämiezeit ist mit schlechteren Ergebnissen bei Lebertransplantationen assoziiert, insbesondere bei Spenderorganen mit einer Makrosteatose $>15 \%[44,45]$ sowie bei Transplantaten älterer Spender [4]. Oliver et al. zeigten kürzlich eine signifikant niedrigere Komplikationsrate in einer ECD-Kohorte mit PLB im Vergleich zu einer Kohorte ohne PLB (31,9 vs. $42,3 \%$; $p<0,001$; [46]). Der positive Einfluss einer standardmäßigen Leberbiopsie auf das Transplantationsergebnis wurde auch schon früher von Markin und Kollegen demonstriert [47]. In dieser Studie wurde die primäre Nichtfunktionsrate von Spenderlebern nach Einführung der PLB von 8,5 auf $1,4 \%$ reduziert.

Darüber hinaus können durch eine PLB unnötige Verwerfungen von Lebertransplantaten vermieden werden, mit damit einhergehender Erweiterung des Pools dringend benötigter Spenderorgane. Bei histologisch diagnostizierter Kontraindikation für eine Transplantation können wiederum unnötige Organentnahmen vermieden werden [46]. Wird eine potenzielle Spenderleber mittels PLB als „marginal“ identifiziert, könnte in ausgewählten Fällen eine Vorkonditionierung (durch maschinelle Perfusion) zur Verbesserung der Transplantatqualität durchgeführt werden [48]. Eine PLB wird in der Regel tagsüber während der regulären Arbeitszeiten durchgeführt, was eine bessere Kommunikation zwischen allen Beteiligten (Pathologe, Transplantationschirurg, DSO) ermöglicht.

Auf der Grundlage der von der Deutschen Stiftung Organtransplantation bereitgestellten Daten konnten wir potenzielle Kosteneinsparungen durch eine
PLB für verschiedene Szenarien abschätzen. Eine genaue Berechnung des Einsparungspotenzials erscheint jedoch schwierig, da dieses von einer Vielzahl verschiedenster Faktoren abhängt, welche sich auf die tatsächlichen Kosten auswirken. Nach unserer Kenntnis hat sich bislang keine Studie mit den möglichen wirtschaftlichen Aspekten der PLB befasst. Hierzu zeigen die $\square$ Abb. 9 und 10 verschiedene Szenarien mit und ohne PLB, jeweils mit möglichen Auswirkungen auf die Kosten während der Allokation. Zusammenfassend belaufen sich die potenziellen Kosteneinsparungen für eine vermeidbare Organentnahme mit anschließendem Organtransport auf bis zu $14.000 €$.

\section{Schlussfolgerung}

Anhand der Ergebnisse unserer Studie konnten wir zeigen, dass eine PLB 1) sicher ist, 2) einen hohen Einfluss auf die Allokation bereits vor der Organentnahme hat (insbesondere bei Spendern mit EDC) und damit in vielen Fällen die kalte Ischämiezeit reduzieren kann, 3) das „matching" zwischen Spender und Empfänger optimiert und 4) ein hohes Kosteneinsparungspotenzial besitzt. Unsere Daten unterstreichen damit die hohe klinische und ökonomische Relevanz der PLB im Rahmen der Lebertransplantation. Unserer Meinung nach sollte eine PLB bei allen potenziellen Spendern, vor allem aber bei solchen mit EDC durchgeführt werden.

\section{Korrespondenzadresse}

\section{Prof. Dr. Silvio Nadalin}

Klinik für Allgemeine, Viszeral- und

Transplantationschirurgie, Universitätsklinikum Tübingen

Tübingen, Deutschland

silvio.nadalin@med.uni-tuebingen.de

Funding. Open Access funding provided by Projekt DEAL.

\section{Einhaltung ethischer Richtlinien}

Interessenkonflikt. C. Beltzer, M. Quante, M. Rheinberger, H.A. Baba, F. Saner, F. Fend, T. Biet, A. Königsrainer und S. Nadalin geben an, dass kein Interessenkonflikt besteht. 
Alle im vorliegenden Manuskript beschriebenen Untersuchungen am Menschen wurden mit Zustimmung der zuständigen Ethikkommission, im Einklang mit nationalem Recht sowie gemäß der Deklaration von Helsinki von 1975 (in der aktuellen, überarbeiteten Fassung) durchgeführt. Einverständniserklärungen wurden von autorisierten Angehörigen aller hirntoten Spender eingeholt.

Open Access. Dieser Artikel wird unter der Creative Commons Namensnennung 4.0 International Lizenz veröffentlicht, welche die Nutzung, Vervielfältigung Bearbeitung, Verbreitung und Wiedergabe in jeglichem Medium und Format erlaubt, sofern Sie den/die ursprünglichen Autor(en) und die Quelle ordnungsgemäß nennen, einen Link zur Creative Commons Lizenz beifügen und angeben, ob Änderungen vorgenommen wurden.

Die in diesem Artikel enthaltenen Bilder und sonstiges Drittmaterial unterliegen ebenfalls der genannten Creative Commons Lizenz, sofern sich aus der Abbildungslegende nichts anderes ergibt. Sofern das betreffende Material nicht unter der genannten Creative Commons Lizenz steht und die betreffende Handlung nicht nach gesetzlichen Vorschriften erlaubt ist, ist für die oben aufgeführten Weiterverwendungen des Materials die Einwilligung des jeweiligen Rechteinhabers einzuholen.

Weitere Details zur Lizenz entnehmen Sie bitte der Lizenzinformation auf http://creativecommons.org/ licenses/by/4.0/deed.de.

\section{Literatur}

1. Durand F, Renz JF, Alkofer B, Burra P, Clavien PA, Porte RJ et al (2008) Report of the Paris consensus meeting on expanded criteria donors in liver transplantation. Liver Transpl 14(12):1694-1707. https://doi.org/10.1002/lt.21668

2. European Association for the Study of the Liver (2016) EASL clinical practice guidelines: liver transplantation. J Hepatol 64(2):433-485. https:// doi.org/10.1016/j.jhep.2015.10.006

3. Blok JJ, Braat AE, Adam R, Burroughs AK, Putter $\mathrm{H}$, Kooreman NG et al (2012) Validation of the donor risk index in orthotopic liver transplantation within the Eurotransplant region. Liver Transpl 18(1):112-119. https://doi.org/10.1002/lt.22447

4. Segev DL, Maley WR, Simpkins CE, Locke JE, Nguyen GC, Montgomery RA et al (2007) Minimizing riskassociated with elderly liver donors by matching to preferred recipients. Hepatology 46(6):1907-1918. https://doi.org/10.1002/hep. 21888

5. Schaubel DE, Sima CS, Goodrich NP, Feng $S$, Merion RM (2008) The survival benefit of deceased donor liver transplantation as a function of candidate disease severity and donor quality. Am J Transplant 8(2):419-425. https://doi.org/10.1111/ j.1600-6143.2007.02086.x

6. Feng S, Goodrich NP, Bragg-Gresham JL, Dykstra DM, Punch JD, DebRoy MA et al (2006) Characteristics associated with liver graft failure: the concept of a donor risk index. Am J Transplant 6(4):783-790. https://doi.org/10.1111/j.16006143.2006.01242.x

7. Braat $A E$, Blok JJ, Putter H, Adam R, Burroughs AK, Rahmel AO et al (2012) The Eurotransplant donor risk index in liver transplantation: ET-DRI. Am J Transplant 12(10):2789-2796. https://doi.org/10. 1111/j.1600-6143.2012.04195.x
8. Schlitt HJ, Loss M, Scherer MN, Becker T, Jauch KW, Nashan B etal (2011) Current developments in liver transplantation in Germany: MELD-based organ allocation and incentives for transplant centres. Z Gastroenterol 49(1):30-38. https://doi.org/10. 1055/s-0029-1245946

9. Savas N, Coskun M, Bilezikci B, Uruc I, Karakayali H, Yilmaz $U$ et al (2008) Value of an individual liver biopsy in the preoperative evaluation of apparently healthy potential liver donors. Liver Transpl 14(4):541-546. https://doi.org/10.1002/lt 21410

10. Rinella ME, Alonso E, Rao S, Whitington P, Fryer J, Abecassis $M$ et al (2001) Body mass index as a predictor of hepatic steatosis in living liver donors. Liver Transpl 7(5):409-414. https://doi. org/10.1053/jlts.2001.23787

11. D'Alessandro AM, Kalayoglu M, Sollinger HW, Hoffmann RM, Reed A, Knechtle SJ et al (1991) The predictive value of donor liver biopsies for the development of primary nonfunction after orthotopic liver transplantation. Transplantation 51(1):157-163

12. Fabbrini E, Sullivan S, Klein S (2010) Obesity and nonalcoholic fatty liver disease: biochemical, metabolic, and clinical implications. Hepatology 51(2):679-689. https://doi.org/10.1002/hep. 23280

13. Dutkowski P, Schlegel A, SlankamenacK, OberkoflerCE, Adam R, Burroughs AKet al (2012) The use of fatty liver grafts in modern allocation systems: risk assessment by the balance of risk (BAR) score. Ann Surg 256(5):861-868. https://doi.org/10.1097/ SLA.0b013e318272dea2 (discussion 868-869)

14. Nocito A, El-Badry AM, Clavien PA (2006) When is steatosis too much for transplantation? J Hepato 45(4):494-499. https://doi.org/10.1016/j.jhep. 2006.07.017

15. Feng S (2008) Steatotic livers for liver transplantation-life-saving but at a cost. Nat Clin Pract Gastroenterol Hepatol 5(7):360-361. https://doi. org/10.1038/ncpgasthep1159

16. Briceno J, Padillo J, Rufian S, Solorzano G, Pera C (2005) Assignment of steatotic livers by the Mayo model for end-stage liver disease. Transpl Int 18(5):577-583. https://doi.org/10.1111/j.14322277.2005.00091.x

17. Ploeg RJ, D'Alessandro AM, KnechtleSJ, Stegall MD, Pirsch JD, Hoffmann RM et al (1993) Risk factors for primary dysfunction after liver transplantation-a multivariate analysis. Transplantation 55(4):807-813

18. Spitzer AL, Lao OB, DickAA, Bakthavatsalam R, Halldorson JB, Yeh MM et al (2010) The biopsied donor liver: incorporating macrosteatosis into high-risk donor assessment. Liver Transpl 16(7):874-884. https://doi.org/10.1002/lt.22085

19. Moosburner S, Gassner J, Nosser M, Pohl J, Wyrwal D, Claussen F et al (2018) Prevalence of steatosis hepatis in the Eurotransplant region: impact on graft acceptance rates. HPB Surg. https://doi.org/10.1155/2018/6094936

20. Tahan V, Canbakan B, Balci H, Dane F, Akin H, Can $G$ et al (2008) Serum gamma-glutamyltranspeptidase distinguishes non-alcoholic fatty liver disease at high risk. Hepatogastroenterology 55(85):1433-1438

21. Franzini M, Fornaciari I, Fierabracci V, Elawadi HA Bolognesi V, Maltinti S et al (2012) Accuracy of b-GGT fraction for the diagnosis of non-alcoholic fatty liver disease. Liver Int 32(4):629-634. https:// doi.org/10.1111/j.1478-3231.2011.02673.x

22. Angulo P, Hui JM, Marchesini G, Bugianesi $E$, George J, Farrell GC et al (2007) The NAFLD fibrosis score: a noninvasive system that identifies liver fibrosis in patients with NAFLD. Hepatology 45(4):846-854. https://doi.org/10.1002/hep. 21496

23. Bedogni G, Miglioli L, Masutti F, Tiribelli C, Marchesini G, Bellentani S (2005) Prevalence of and risk factors for nonalcoholic fatty liver disease: the Dionysos nutrition and liver study. Hepatology 42(1):44-52. https://doi.org/10.1002/hep.20734

24. Neuschwander-Tetri BA, Caldwell SH (2003) Nonalcoholic steatohepatitis: summary of an AASLD single topic conference. Hepatology 37(5):1202-1219. https://doi.org/10.1053/jhep. 2003.50193

25. Adani GL, Baccarani U, Sainz-Barriga M, Lorenzin D, Bresadola V, Risaliti A et al (2006) The role of hepatic biopsy to detect macrovacuolar steatosis during liver procurement. Transplant Proc 38(5):1404-1406. https://doi.org/10.1016/j. transproceed.2006.02.111

26. Ma X, Holalkere NS, Kambadakone RA, Mino-Kenudson M, Hahn PF, Sahani DV (2009) Imaging-based quantification of hepatic fat: methods and clinical applications. Radiographics 29(5):1253-1277. https://doi.org/10.1148/ rg. 295085186

27. Frankel WL, Tranovich JG, Salter L, Bumgardner G, BakerP(2002) Theoptimal number of donorbiopsy sites to evaluate liver histology for transplantation. Liver Transpl 8(11):1044-1050. https://doi.org/10. 1053/jlts.2002.36492

28. Silva MA (2009) Putting objectivityinto assessment of steatosis. Transplantation 88(5):620-621. https://doi.org/10.1097/TP.0b013e3181b39085

29. Cesaretti M, Addeo P, Schiavo L, Anty R, lannelli A (2019) Assessment of liver graft steatosis: Where do we stand? Liver Transpl 25(3):500-509. https:// doi.org/10.1002/lt.25379

30. Schleicher C, KreipeHH, SchemmerP, Strassburg CP, Fischer-Frohlich CL, Rahmel A et al (2019) Donor liver histology: joint recommendations of the DGP, DTG and DSO. Chirurg 90(11):899-904. https://doi. org/10.1007/s00104-019-0990-5

31. Imber CJ, St Peter SD, Lopez I, Guiver L, Friend PJ (2002) Current practice regarding the use of fatty livers: a trans-Atlantic survey. Liver Transpl 8(6):545-549. https://doi.org/10.1053/jlts.2002. 31747

32. Oliver JB, Marcus AF, Paster M, Nespral J, Bongu A, Dikdan $G$ et al (2017) Organ procurement organization survey of practices and beliefs regarding prerecovery percutaneous liver biopsy in donation after neurologic determination of death. Transplantation 101(4):821-825. https:// doi.org/10.1097/TP.0000000000001632

33. Nadalin S, Malago M, Valentin-Gamazo C, Testa G, Baba HA, Liu Cet al (2005) Preoperative donor liver biopsy for adult living donor liver transplantation: risks and benefits. Liver Transpl 11(8):980-986. https://doi.org/10.1002/lt.20462

34. Moosburner S, Sauer IM, Gassner J, Schleicher C, BosebeckD, Rahmel A et al (2019) Macrosteatosis is a huge problem in liver transplantation-however, not the only one we face. Am J Transplant 19(9):2661-2662. https://doi.org/10.1111/ajt. 15418

35. Ryan CK, Johnson LA, Germin BI, Marcos A (2002) One hundred consecutive hepatic biopsies in the workup of living donors for right lobe liver transplantation. Liver Transpl 8(12):1114-1122. https://doi.org/10.1053/jlts.2002.36740

36. Cadranel JF, Rufat P, Degos F (2000) Practices of liver biopsy in France: results of a prospective nationwide survey. For the Group of Epidemiology 


\section{Originalien}

of the French Association for the Study of the Liver (AFEF). Hepatology 32(3):477-481. https:// doi.org/10.1053/jhep.2000.16602

37. Westheim BH, Aagenæs I, Østensen AB, Sanengen T, Almaas R (2013) Effect of operator experience and frequency of procedure performance on complication rate after ultrasoundguided percutaneous liver biopsies. J Pediatr Gastroenterol Nutr 57(5):638-643. https://doi. org/10.1097/MPG.0b013e3182a0c7a5

38. Mueller M, Kratzer W, Oeztuerk S, Wilhelm M, Mason RA, Mao R et al (2012) Percutaneous ultrasonographically guided liver punctures: an analysis of 1961 patients over a period of ten years. BMC Gastroenterol 12:173. https://doi.org/ 10.1186/1471-230x-12-173

39. Tannapfel A, Dienes H-P, Lohse AW (2012) The indications for liver biopsy. Dtsch Arztebl Int 109(27-28):477

40. Adam R, Reynes M, Johann M, Morino M, Astarcioglu I, Kafetzis I et al (1991) The outcome of steatotic grafts in liver transplantation. Transplant Proc 23(1 Pt 2):1538-1540

41. Chavin KD, Fiorini RN, Shafizadeh S, Cheng G, Wan C, Evans Z et al (2004) Fatty acid synthase blockade protects steatotic livers from warm ischemia reperfusion injury and transplantation. Am J Transplant 4(9):1440-1447. https://doi.org/ 10.1111/j.1600-6143.2004.00546.x

42. Czerwiński J, Perkowska A, Mróz A, Lagiewska B, Adadyński L, Durlik M et al (2007) Assessment of cadaveric livers discarded from transplantation. A correlation between clinical and histological parameters. Ann Transplant 12(2):30-36
43. Yersiz H, Lee C, Kaldas FM, Hong JC, Rana A, Schnickel GT etal (2013) Assessment of hepatic steatosis by transplant surgeon and expert pathologist: a prospective, double-blind evaluation of 201 donor livers. Liver Transpl 19(4):437-449. https:// doi.org/10.1002/lt.23615

44. Salizzoni M, Franchello A, Zamboni F, Ricchiuti A, Cocchis D, FopFetal (2003) Marginal grafts: finding the correct treatment for fatty livers. Transpl Int 16(7):486-493. https://doi.org/10.1007/s00147003-0572-8

45. Fukumori T, Ohkohchi N, Tsukamoto S, Satomi S (1999) The mechanism of injury in a steatotic live graft during cold preservation. Transplantation 67(2):195-200

46. Oliver JB, Machineni P, Bongu A, Patel T, Nespral J, Kadric $C$ et al (2018) Liver biopsy in assessment of extended criteria donors. Liver Transpl 24(2):182-191. https://doi.org/10.1002/lt.24947

47. Markin RS, Wisecarver JL, Radio SJ, Stratta RJ, Langnas AN, Hirst K et al (1993) Frozen section evaluation of donor livers before transplantation. Transplantation 56(6):1403-1409

48. Karimian N, Yeh H (2017) Opportunities for therapeutic intervention during machine perfusion. Curr Transpl Rep 4(2):141-148. https://doi.org/10 1007/s40472-017-0144-y

\section{Hier steht eine Anzeige.}

\section{黑 Springer}


Hier steht eine Anzeige.

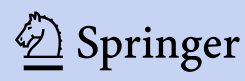

